\title{
Building a Kinetic Model of Trehalose BIOSYNTHESIS IN SACCHAROMYCES
}

CEREVISIAE

Kieran Smallbone, Naglis Malys, Hanan L. Messiha, Jill

A. Wishart, and Evangelos Simeonidis

\section{Contents}

1. Introduction 356

2. Biological Background $\quad 357$

2.1. T6P synthase complex 358

2.2. Response to stress 358

2.3. Interaction with glycolysis $\quad 360$

3. Model Development 363

3.1. Hexokinase 363

3.2. Phosphoglucomutase 364

3.3. UDP-glucose phosphorylase $\quad 364$

3.4. T6P synthase 364

3.5. T6P phosphatase $\quad 365$

3.6. Trehalase 365

3.7. Glucose transport $\quad 365$

3.8. G6P isomerase 365

3.9. $V_{\max } \quad 365$

3.10. Systems biology standards 366

4. Results 366

4.1. Heat shock 366

4.2. TPS1 mutant 366

5. Discussion 367

$\begin{array}{ll}\text { Acknowledgments } & 368\end{array}$

$\begin{array}{ll}\text { References } & 368\end{array}$

Manchester Centre for Integrative Systems Biology, The University of Manchester, Manchester, United 


\begin{abstract}
In this chapter, we describe the steps needed to create a kinetic model of a metabolic pathway based on kinetic data from experimental measurements and literature review. Our methodology is presented by utilizing the example of trehalose metabolism in yeast. The biology of the trehalose cycle is briefly reviewed and discussed.
\end{abstract}

\title{
1. INTRODUCTION
}

The emergent field of systems biology involves the study of the interactions between the components of a biological system and how these interactions give rise to the function and behavior of this system (e.g., the enzymes and metabolites in a metabolic pathway). Nonlinear processes dominate the dynamic behavior of such biological networks, and hence intuitive verbal reasoning approaches are insufficient to describe the resulting complex system dynamics (Lazebnik, 2002; Mendes and Kell, 1998; Szallasi et al., 2006). Nor can such approaches keep pace with the large increases in -omics data (such as metabolomics and proteomics) and the accompanying advances in high-throughput experiments and bioinformatics. Rather, experience from other areas of science has taught us that quantitative methods are needed to develop comprehensive theoretical models for interpretation, organization, and integration of this data. Once viewed with scepticism, we now realize that mathematical models, continuously revised to incorporate new information, must be used to guide experimental design and interpretation.

We focus here on mathematical models of cellular metabolism (Klipp et al., 2005; Palsson, 2006; Wiechert, 2002). In recent years, two major (and divergent) modeling methodologies have been adopted to increase our understanding of metabolism and its regulation. The first is constraint-based modeling (Covert et al., 2003; Price et al., 2004), which uses physicochemical constraints such as mass balance, energy balance, and flux limitations to describe the potential behavior of an organism. The biochemical structure of (at least the central) metabolic pathways is more or less well known, and hence the stoichiometry of such a network may be deduced. In addition, the flux of each reaction through the system may be constrained through, for example, knowledge of its $V_{\max }$, or irreversibility considerations. From the steady-state solution space of all possible fluxes, a number of techniques have been proposed to deduce network behavior, including flux balance and extreme pathway or elementary mode analysis. In particular, flux balance analysis (FBA) (Kauffman et al., 2003) highlights the most effective and efficient paths through the network in order to achieve a particular objective function, such as the maximization of biomass or ATP production. 
The key benefit of FBA lies in the minimal amount of biological knowledge and data required to make quantitative inferences about network behavior. This apparent "free lunch" comes at a price, as constraintbased modeling is concerned only with fluxes through the system and makes neither inferences nor predictions about cellular metabolite concentrations. By contrast, kinetic modeling aims to characterize the mechanics of each enzymatic reaction, in terms of how changes in metabolite concentrations affect local reaction rates. However, a considerable amount of data is required to parameterize a mechanistic model; if complex reactions like phosphofructokinase are involved, an enzyme kinetic formula may have 10 or more kinetic parameters (Wiechert, 2002). The determination of such parameters is costly and time consuming, and moreover, many may be difficult or impossible to determine experimentally. The in vivo molecular kinetics of some important processes like oxidative phosphorylation and many transport mechanisms are almost completely unknown, so that modeling assumptions about these metabolic processes are necessarily highly speculative.

Because precise kinetic formulas are missing for many enzymes, simplified or phenomenological approaches are used frequently to facilitate modeling. One well-known approach is the power law formalism which uses an exponential expression for each reaction step (Voit, 1991). Alternatives include linlog kinetics, which draws ideas from thermodynamics and metabolic control analysis (Hatzimanikatis and Bailey, 1997; Smallbone et al., 2007) and convenience kinetics, which provides a more realistic approximation to the underlying enzymatic mechanisms (Liebermeister and Klipp, 2006). Finally, thermodynamic flow-force relationships are a way to relate thermodynamics to kinetics (Westerhoff and van Dam, 1987).

In this chapter, we describe the steps needed to create a kinetic model of a metabolic pathway. We use trehalose metabolism in yeast as an example to apply the methodology required for this purpose. But before addressing the mathematics, we need to understand the biology underlying the system.

\section{BiOLOGICAL BACKGROUND}

The view of the role of trehalose, extensively studied in baker's yeast, has changed over the years. Its apparent role is to function as a carbohydrate reservoir, but it has now gained new importance as a crucial part of a stabilizing mechanism for proteins and cellular membranes under stress conditions such as heat shock (Crowe et al., 1984; Singer and Lindquist, 1998). Under a stress environment, Saccharomyces cerevisiae is able to increase the concentration of trehalose up to $15 \%$ of cell dry mass (François and Parrou, 2001). The metabolic pathway that produces trehalose is believed to 
regulate glucose uptake, particularly when the cell exists in an adverse environment. For the above reasons, and also because of its numerous applications in the cosmetics, food, and pharmaceutical industries, trehalose has become an important biotechnological product. It has also been found that trehalose 6-phosphate (T6P), an intermediate of trehalose biosynthesis, plays a key role in the control of glycolytic flux (Blázquez et al., 1993).

Trehalose is a disaccharide synthesized from two glucose subunits through a pathway elucidated in yeast over 50 years ago (Cabib and Leloir, 1958). The trehalose pathway in yeast consists of a small number of reactions, but these are "arranged" in a metabolic cycle and are governed by a highly complicated regulatory system of inhibitions and/or activations. Due to this complexity, the operation of the pathway is difficult to study experimentally. Glucose is converted into glucose 6-phosphate (G6P), which, together with uridine diphosphate (UDP) glucose, leads to the formation of T6P and, subsequently, trehalose. Trehalose can, in turn, be hydrolyzed into two glucose molecules, thereby closing the trehalose cycle (see Fig. 18.1).

\subsection{T6P synthase complex}

In $S$. cerevisiae, the enzymes that catalyze the reactions of trehalose biosynthesis, T6P synthase (TPS1) and T6P phosphatase (TPS2), form a complex with two other stabilizing, noncatalytic proteins (TSL1 and TPS3). While this complex provides the primary mechanism for trehalose biosynthesis, TPS1 can function independently of the other units of the complex and T6P can be dephosphorylated by other (unspecific) phosphatases (Bell et al., 1998). An important property of this protein complex is its strong temperature activation, with an optimum at around $45^{\circ} \mathrm{C}$.

T6P synthase activity for the complex has affinities for G6P and UDP glucose higher than their concentration in the cells (see Tables 18.1 and 18.2). The enzyme is also strongly noncompetitively inhibited by phosphate. Accordingly, the rate of trehalose synthesis is heavily influenced by changes in both substrate concentration and temperature.

\subsection{Response to stress}

Cells subjected to stress respond with an interplay of transcriptional and posttranslational changes that lead to a stress resistant state. Trehalose belongs to the early metabolic response, as exposure to various stresses leads to a rapid increase in its concentration. Its primary role is to protect membranes from desiccation, though it can also protect proteins from denaturation in hydrated cells.

In yeast, the transcription factors Msn2p and Msn4p control an environmental stress response (Berry and Gasch, 2008). Upon heat, osmotic shock, oxidative stress, and nutrient starvation, Msn2p/Msn4p are phosphorylated 


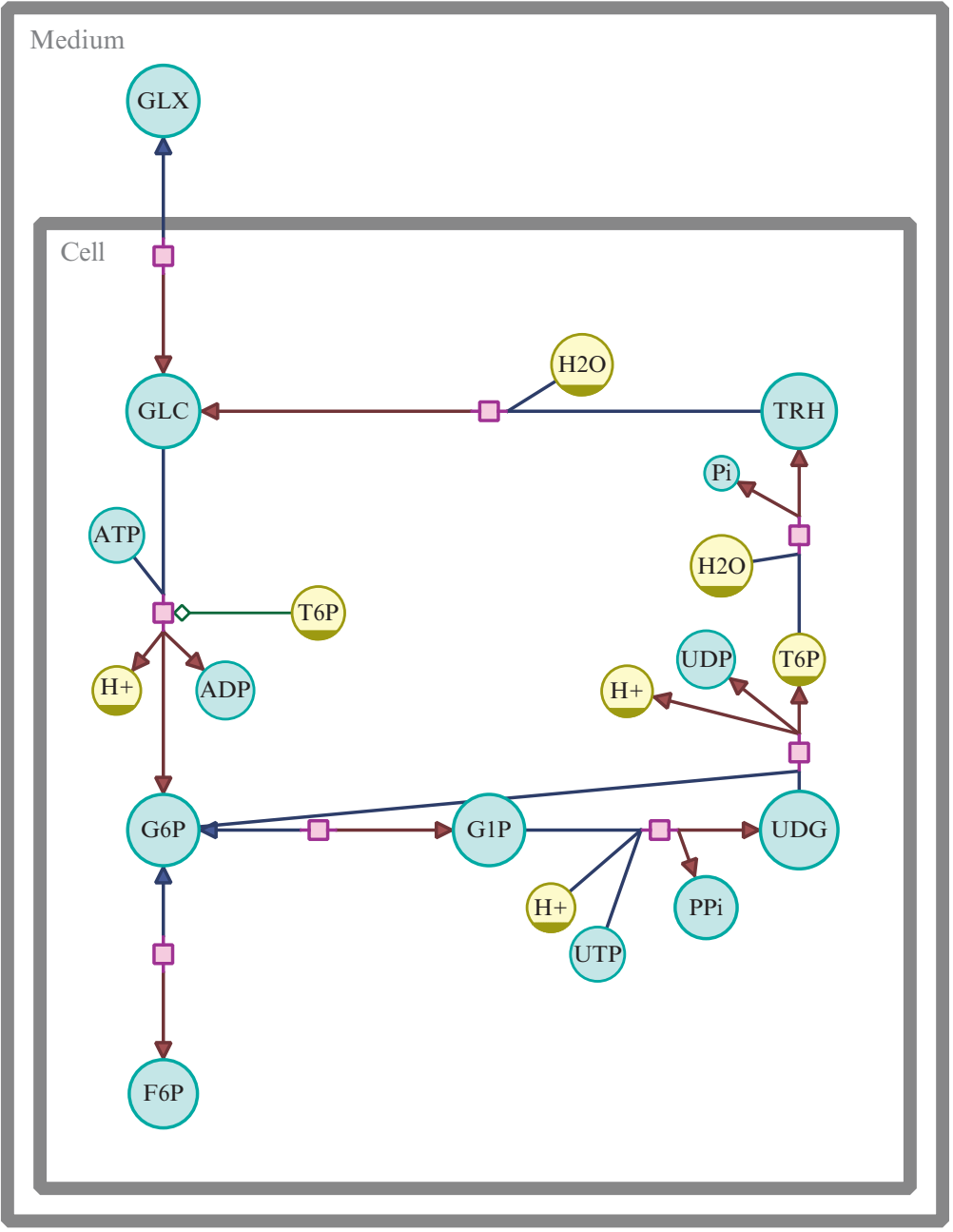

Figure 18.1 The trehalose cycle. Diagram created in Arcadia (Villéger et al., 2010) and made available in Systems Biology Graphical Notation (SBGN, Le Novère et al., 2009) format. Reactive metabolites are marked with a blue circle, ubiquitous metabolites with a yellow circle, reactions with a square, and regulators with a diamond. GLX, external glucose; GLC, glucose; G6P, glucose 6-phosphate; F6P, fructose 6-phosphate; G1P, glucose 1-phosphate; UTP, uridine triphosphate; UDG, UDP-glucose; PPi, diphosphate; T6P, trehalose 6-phosphate; UDP, uridine diphosphate; TRH, trehalose.

by protein kinase A and translocated to the nucleus. Here, these factors bind to the stress response element (STRE) in gene promoters stimulating the expression of a large group of stress response genes. 
Table 18.1 Metabolite concentrations used in the model

\begin{tabular}{|lll|}
\hline Metabolite & Concentration $(\mathrm{mM})$ & Reference \\
ADP & 1.282 & Pritchard and Kell (2002) \\
ATP & 2.525 & Pritchard and Kell (2002) \\
Fructose 6-phosphate & 0.625 & Pritchard and Kell (2002) \\
Glucose (extracellular) & 100 & Pritchard and Kell (2002) \\
Glucose (intracellular) & 0.09675 & Pritchard and Kell (2002) \\
Glucose 1-phosphate & 0.1 & Voit (2003) \\
Glucose 6-phosphate & 2.675 & Pritchard and Kell (2002) \\
Trehalose & 0.05 & Voit $(2003)$ \\
Trehalose 6-phosphate & 0.02 & Voit (2003) \\
UDP & 0.2815 & - \\
UDP glucose & 0.7 & Voit (2003) \\
UTP & 0.6491 & - \\
\hline
\end{tabular}

$[\mathrm{UDP}]$ and $[\mathrm{UTP}]$ are calculated from $[\mathrm{ATP}]$ using ratios ATP:UDP and ATP:UTP of 8.97 and 3.89, respectively (Canelas et al., 2009).

The two best-characterized cases of response to stress-temperature upshift and osmotic shock - show the induction of a wide class of genes including key players of carbohydrate and trehalose metabolism, in particular (Table 18.3). In the case of heat shock, cells subjected to a temperature of $33-38{ }^{\circ} \mathrm{C}$ experience a complicated set of changes; the slight accumulation of trehalose is primarily due to transcriptional activation of T6P1. However, if cells are subjected to acute heat shock (over $40{ }^{\circ} \mathrm{C}$ ), the induction of STRE-containing genes is totally abolished. Rather, the efficacy to accumulate trehalose at high temperature is a consequence of both direct stimulation of T6P synthase complex activity and inhibition of trehalase activity (François and Parrou, 2001).

In addition to environmental stress responses, genes involved in trehalose metabolism can be induced if other gene functions are deficient, for example, scavenger decapping pyrophosphatase (De Mesquita et al., 2003; Malys et al., 2004).

\subsection{Interaction with glycolysis}

An unexpected link between the trehalose and glycolytic pathways is that mutations in TPS1 prevent cellular growth on glucose (Thevelein and Hohmann, 1995). Within a few seconds of sugar addition, TPS1 mutants accumulate large amounts of hexose monophosphates and fructose 1,6bisphosphate and show a depletion of ATP and a reduction of intracellular phosphate. This reveals an imbalance between the upper (ATP consuming) and lower (ATP regenerating) parts of glycolysis. The imbalance must be 
Table 18.2 Parameter values used in the model

\begin{tabular}{|c|c|c|}
\hline Parameter & Value & Reference \\
\hline \multicolumn{3}{|l|}{ Hexokinase } \\
\hline$K_{\mathrm{GLC}}$ & $0.08 \mathrm{mM}$ & Pritchard and Kell (2002) \\
\hline$K_{\text {ATP }}$ & $0.15 \mathrm{mM}$ & Pritchard and Kell (2002) \\
\hline$K_{\mathrm{G} 6 \mathrm{P}}$ & $30 \mathrm{mM}$ & Pritchard and Kell (2002) \\
\hline$K_{\mathrm{ADP}}$ & $0.23 \mathrm{mM}$ & Pritchard and Kell (2002) \\
\hline$K_{\text {iTRH }}$ & $0.04 \mathrm{mM}$ & Blázquez et al. (1993) \\
\hline$K_{\mathrm{eq}}$ & 2000 & Pritchard and Kell (2002) \\
\hline \multicolumn{3}{|c|}{ Phosphoglucomutase } \\
\hline$K_{\mathrm{G} 6 \mathrm{P}}$ & $0.05 \mathrm{mM}$ & Ray and Roscelli (1964) \\
\hline$K_{\mathrm{G} 1 \mathrm{P}}$ & $0.023 \mathrm{mM}$ & Daugherty et al. (1975) \\
\hline$K_{\mathrm{eq}}$ & $1 / 6$ & Wright et al. (1977) \\
\hline \multicolumn{3}{|c|}{ UDP-glucose phosphorylase } \\
\hline$K_{\mathrm{UTP}}$ & $0.11 \mathrm{mM}$ & Wright et al. (1977) \\
\hline$K_{\mathrm{iUTP}}$ & $0.11 \mathrm{mM}$ & Wright et al. (1977) \\
\hline$K_{\mathrm{G} 1 \mathrm{P}}$ & $0.32 \mathrm{mM}$ & Wright et al. (1977) \\
\hline$K_{\mathrm{iUDG}}$ & $0.035 \mathrm{mM}$ & Guranowski et al. (2004) \\
\hline \multicolumn{3}{|c|}{ T6P synthase } \\
\hline$K_{\mathrm{G} 6 \mathrm{P}}$ & $3.8( \pm 0.57) \mathrm{mM}$ & MCISB \\
\hline$K_{\mathrm{UDPG}}$ & $0.886( \pm 0.16) \mathrm{mM}$ & MCISB \\
\hline \multicolumn{3}{|c|}{ T6P phosphatase } \\
\hline $\begin{array}{l}K_{\mathrm{T} 6 \mathrm{P}} \\
\text { Trehalase }\end{array}$ & $0.5 \mathrm{mM}$ & Vandercammen et al. (1989) \\
\hline$K_{\mathrm{TRE}}$ & $2.99( \pm 0.45) \mathrm{mM}$ & MCISB \\
\hline
\end{tabular}

MCISB enzymes were expressed in S. cerevisiae overexpression strains (Gelperin et al., 2005), purified, and quantified as described in Malys et al. (2010).

rectified in wild-type yeast either through a decrease in the rate of the initial glycolytic steps or through an increase in substrates (such as free phosphate) to activate the second part of glycolysis.

The primary explanation for the observed phenotype of TPS1 mutants is that control is provided by T6P, which competitively inhibits hexokinase 2 at submillimolar concentrations (Blázquez et al., 1993). But this is rather implausible; one would expect T6P to be channeled within the protein complex synthesizing trehalose and "it appears unlikely that the massive flux through yeast glycolysis would be entirely dependent on fortuitous seeping of T6P from the complex" (Thevelein and Hohmann, 1995). Moreover, a 50-fold overexpression of hexokinase 2 does not lead to a TPS1 null phenotype.

T6P inhibition of hexokinase is not the complete picture of glycolysis regulation by the trehalose cycle. A number of alternatives have been proposed to explain the TPS1 phenotype (Gancedo and Flores, 2004). 
Table 18.3 Changes in enzymatic activities and transport steps induced by heat and osmotic shock

\begin{tabular}{|lll|}
\hline & \multicolumn{2}{l|}{ Fold change in activity upon } \\
\cline { 2 - 3 } Reaction & Heat shock & Osmotic shock \\
Glucose transport & 8 & 9 \\
Hexokinase & 8 & 15 \\
G6P isomerase & 1 & 1 \\
Phosphoglucomutase & 16 & 5 \\
UDP-glucose phosphorylase & 16 & 11 \\
T6P synthase & 12 & 6 \\
T6P phosphatase & 18 & 20 \\
Trehalase & 6 & 8 \\
\hline
\end{tabular}

Heat shock data from Voit (2003) (processed from the transcriptomics data of Gasch et al., 2000) and osmotic shock data from Rep et al. (2000).

It has been suggested that trehalose synthase could recycle inorganic phosphate to avoid a blockage of glycolysis at the glyceraldehyde 3-phosphate dehydrogenase step; but this seems unlikely as glycolysis flux is a 100 -fold faster than trehalose formation, making it inconceivable that the pathway could recycle sufficient phosphate. A second proposal suggested that TPS1, hexokinase, and the glucose carrier could form a glucose-sensing complex, but this is very much hypothetical.

It has been suggested that trehalose turnover may function as a glycolytic safety valve (Blomberg, 2000). Comparing glycolysis to the turbo design of a motor, it is predicted that lack of a control mechanism results in a steady rise of sugar phosphates until ATP and phosphates are depleted (Teusink et al., 1998). The recycling of trehalose via a futile ATP cycle could avoid substrate-accelerated death under stress.

Recent evidence suggests that the TPS1 gene product modulates mitochondrial respiratory content through cAMP and interaction with hexokinase 2 (Noubhani et al., 2009). Assuming this is correct, it makes sense physiologically that both glycolysis and mitochondrial activity are controlled by a common regulatory mechanism, in which when the glycolysis flux is inhibited, the mitochondrial respiration can be activated allowing the balancing of ATP between glycolysis and oxidative respiration.

One experimental feature not explained by any of the above hypotheses is the lack of fermentation in TPS1 mutants, despite their accumulation of hexose phosphates. An interesting suggestion is that the second half of glycolysis is activated by T6P or TPS1. But, again, this is hypothetical, and the only experimentally demonstrated connection found between trehalose biosynthesis and glycolysis is the inhibition of hexokinase by T6P. 


\section{Model DeVelopment}

Despite its small size, the trehalose cycle is governed by a surprisingly complex control mechanism, and its interaction with glycolysis is poorly understood. Intuitive, verbal reasoning approaches are insufficient to describe the resulting complex system dynamics. Rather, quantitative methods are needed to develop comprehensive theoretical models for interpretation, organization, and integration of available data. We present here a methodology for building a kinetic model of the trehalose cycle, based on characterizations of the mechanism of each enzymatic reaction in the pathway. The model's kinetic constants were collected through experimentation, literature review, and with the help of a text-mining toolbox, KiPar, developed to retrieve kinetic parameters of interest from publicly available scientific literature (Spasić et al., 2009).

A mathematical description of a kinetic metabolic model may be given in differential equation form as

$$
x^{\prime}=N v(x, y, p), \quad x(0)=x_{0},
$$

and this may be used as a guide as to the data required to create and parameterize a kinetic model.

First, $N$ is the stoichiometric matrix, which may be derived easily from the topology of the model (Fig. 18.1) - here derived from a recent genomescale reconstruction of yeast metabolism (Dobson et al., 2010). Symbol $x$ denotes metabolite concentrations, which for our pathway are the concentrations of glucose, G6P, glucose 1-phosphate, UDP glucose, T6P, and trehalose. Symbol $y$ denotes boundary metabolites, whose concentrations are not allowed to vary but do affect the reaction rates; in our case, these are ADP, ATP, fructose 6-phosphate, extracellular glucose, UDP, and UTP. Concentrations for both $x$ and $y$ must be defined (see Table 18.1), though note that only concentrations $x$ will change over time. Finally, $v$ denotes reaction rates; these are dependent on kinetic mechanisms, concentrations $\left(x\right.$ and $y$ ), and parameters $(p)$ - typically $V_{\max }$ and $K_{\mathrm{m}}$. Further information on the individual reactions is given below. Parameter values may be found in Table 18.2.

\subsection{Hexokinase}

$$
\text { Glucose }+\mathrm{ATP} \rightarrow \text { glucose 6-phosphate }+\mathrm{ADP}+\mathrm{H}^{+}
$$

Following Pritchard and Kell (2002), hexokinase is modeled using bi-bi kinetics; in addition, we allow for the competitive inhibition of glucose by T6P: 


$$
\frac{v}{V_{\max }}=\frac{\frac{1}{K_{\mathrm{GLC}} K_{\mathrm{ATP}}}\left([\mathrm{GLC}][\mathrm{ATP}]-\frac{[\mathrm{G} 6 \mathrm{P}][\mathrm{ADP}]}{K_{\mathrm{eq}}}\right)}{\left(1+\frac{[\mathrm{GLC}]}{K_{\mathrm{GLC}}}+\frac{[\mathrm{G} 6 \mathrm{P}]}{K_{\mathrm{G} 6 \mathrm{P}}}+\frac{[\mathrm{T} 6 \mathrm{P}]}{K_{\mathrm{T} 6 \mathrm{P}}}\right)\left(1+\frac{[\mathrm{ATP}]}{K_{\mathrm{ATP}}}+\frac{[\mathrm{ADP}]}{K_{\mathrm{ADP}}}\right)}
$$

\subsection{Phosphoglucomutase}

Glucose 6-phosphate $\leftrightarrow$ glucose 1-phosphate

Phosphoglucomutase is modeled using uni-uni kinetics:

$$
\frac{v}{V_{\max }}=\frac{\frac{1}{K_{\mathrm{G} 6 \mathrm{P}}}\left([\mathrm{G} 6 \mathrm{P}]-\frac{[\mathrm{G} 1 \mathrm{P}]}{K_{\mathrm{eq}}}\right)}{1+\frac{[\mathrm{G} 6 \mathrm{P}]}{K_{\mathrm{G} 6 \mathrm{P}}}+\frac{[\mathrm{G} 1 \mathrm{P}]}{K_{\mathrm{G} 1 \mathrm{P}}}} .
$$

\subsection{UDP-glucose phosphorylase}

Glucose 1-phosphate $+\mathrm{UTP}+\mathrm{H}^{+} \rightarrow$ UDP glucose + diphosphate

Following Wright et al. (1977), this is modeled using an ordered bi-bi mechanism:

$$
\frac{v}{V_{\max }}=\frac{\frac{[\mathrm{UTP}]}{K_{\mathrm{UTP}}} \frac{[\mathrm{G} 1 \mathrm{P}]}{K_{\mathrm{G} 1 \mathrm{P}}}}{\frac{K_{\mathrm{UUTP}}}{K_{\mathrm{UTP}}}+\frac{[\mathrm{UTP}]}{K_{\mathrm{UTP}}}+\frac{[\mathrm{G} 1 \mathrm{P}]}{K_{\mathrm{G} 1 \mathrm{P}}}+\frac{[\mathrm{UTP}]}{K_{\mathrm{UTP}}} \frac{[\mathrm{G} 1 \mathrm{P}]}{K_{\mathrm{G} 1 \mathrm{P}}}+\frac{K_{\mathrm{iUTP}}}{K_{\mathrm{UTP}}} \frac{[\mathrm{UDPG}]}{K_{\mathrm{iUDP}}}+\frac{[\mathrm{G} 1 \mathrm{P}]}{K_{\mathrm{G} 1 \mathrm{P}}} \frac{[\mathrm{UDPG}]}{K_{\mathrm{UUDP}}}} .
$$

The product inhibition expression used does not include inhibition constants for diphosphate, as this product is assumed to be effectively hydrolyzed in vivo.

\subsection{T6P synthase}

$$
\begin{array}{r}
\text { Glucose 6-phosphate + UDP glucose } \\
\rightarrow \text { trehalose 6-phosphate }+\mathrm{UDP}+\mathrm{H}^{+}
\end{array}
$$

This reaction is irreversible (Cabib and Leloir, 1958). Given this irreversibility and no information regarding the enzymatic mechanism, or product inhibition levels, we assume the reaction to be uninhibited by its products:

$$
\frac{v}{V_{\max }}=\frac{\frac{[\mathrm{G} 6 \mathrm{P}]}{K_{\mathrm{G} 6 \mathrm{P}}} \frac{[\mathrm{UDPG}]}{K_{\mathrm{UDPG}}}}{\left(1+\left[\frac{\mathrm{G} 6 \mathrm{P}]}{K_{\mathrm{G} G \mathrm{P}}}\right)\left(1+\frac{[\mathrm{UDPG}]}{K_{\mathrm{UDPG}}}\right)\right.}
$$


It is clear that such a form will not capture the complexities of this step discussed above. Nonetheless, use of simple kinetics is most appropriate in the absence of clear experimental guidance.

\subsection{T6P phosphatase}

Trehalose 6-phosphate + water $\rightarrow$ trehalose + phosphate

T6P phosphatase is not inhibited by trehalose (Vandercammen et al., 1989), so we model the reaction as

$$
\frac{v}{V_{\max }}=\frac{\frac{[\mathrm{T} 6 \mathrm{P}]}{K_{\mathrm{T} 6 \mathrm{P}}}}{1+\frac{[\mathrm{T} 6 \mathrm{P}]}{K_{\mathrm{T} 6 \mathrm{P}}}} .
$$

\subsection{Trehalase}

$$
\text { Trehalose }+ \text { water } \rightarrow 2 \text { glucose }
$$

Trehalase is not inhibited by glucose (Wright et al., 1977), so we model the reaction as

$$
\frac{v}{V \max }=\frac{\frac{[\mathrm{TRE}]}{K_{\mathrm{TRE}}}}{1+\frac{[\mathrm{TRE}]}{K_{\mathrm{TRE}}}} .
$$

Two reactions were modeled as per Pritchard and Kell (2002):

\subsection{Glucose transport}

$$
\text { Glucose[medium] } \leftrightarrow \text { glucose[cell] }
$$

\subsection{G6P isomerase}

Glucose 6-phosphate $\leftrightarrow$ fructose 6-phosphate

\section{9. $V_{\max }$}

$V_{\max }$ parameters have not been defined in Table 18.2. Such parameters typically vary wildly, even within a single species, and are highly dependent upon growth conditions. However, reaction fluxes through glycolysis (89.33 mM/min; Pritchard and Kell, 2002) and trehalose $(0.5 \mathrm{mM} / \mathrm{min}$; 
Voit, 2003) are known. Using these fluxes and the other known parameter values, $V_{\max }$ values may be inferred.

\subsection{Systems biology standards}

Describing mathematical models as above is unwieldy and error-prone and naturally leads to difficulties in reproduction of results. Thus researchers have developed SBML (the Systems Biology Markup Language, Hucka et al., 2003), a computer-readable format, for representing models of biological processes that is supported by many software packages. SBML can be combined with MIRIAM (the Minimum Information Requested In the Annotation of biochemical Models, Le Novère et al., 2005) to annotate the entities of those models, for example, by marking-up the molecule "GLC" as CHEBI:17925 (http://www.ebi.ac.uk/chebi/ searchId.do?chebiId=CHEBI:17925) allows its unambiguous identification and automatically links to many additional sources of information. For more information on these standards, see Krause et al. (2010).

The SBML model is available from BioModels.net (Li et al., 2010), a modeling repository (see http://www.ebi.ac.uk/biomodels-main/MODEL 1010010000).

\section{ReSULtS}

\subsection{Heat shock}

Response of the cell to heat shock is simulated by modifying the enzymatic activities and transport steps by the factors defined in Table 18.3. Expression of genes involved in trehalose turnover is stimulated under many types of environmental change. However, increase in production of trehalose is only measurable under specific conditions (e.g., heat shock). This suggests that the mRNA is not always translated into protein (and active enzyme). Moreover, the level of protein synthesis does not always correlate with transcription. Therefore, the increase in mRNA level and the increase in protein activity are incorporated into these values.

Results are presented in Table 18.4: we see a large increase in the levels of G6P and trehalose. This is in agreement with the previously measured 10- to 17-fold increase in trehalose when cells were grown under heat shock conditions at $36^{\circ} \mathrm{C}$ (Hottiger et al., 1987; Ribeiro et al., 1994).

\subsection{TPS1 mutant}

The effects of knocking out TPS1 are simulated by reducing TPS1 activity to $1 \%$ of its normal level. Results are presented in Table 18.4: we see a build-up of some intermediates in the cycle (G6P and UDP glucose) and a 
Table 18.4 Response of model concentrations to heat shock and TPS1 mutation

\begin{tabular}{|lll|}
\hline Metabolite & Heat shock & TPS1 mutant \\
Glucose & 0.121 & -0.146 \\
Glucose 6-phosphate & 0.728 & 0.00213 \\
Glucose 1-phosphate & 0.0787 & 0.642 \\
UDP glucose & -0.0636 & 2.05 \\
Trehalose 6-phosphate & 0.0718 & -1.67 \\
Trehalose & 0.565 & -1.66 \\
\hline
\end{tabular}

Results are presented as $\log \left([\mathrm{X}]_{\mathrm{ss}} /[\mathrm{X}]_{0}\right)$, so a value of 2 refers to a $10^{2}=100$-fold increase in concentration.

drop in concentration of others (T6P and trehalose). A minor increase in G6P is observed, suggesting that the 50-fold decrease in T6P concentration (from 20 to $0.4 \mu \mathrm{M}$ ) may only have a minor affect on the hexokinase activity (the observed $K_{i}$ of T6P on hexokinase 1 and hexokinase 2 are 200 and $40 \mu \mathrm{M}$, respectively, Blázquez et al., 1993). This further questions the possibility of the inhibition of hexokinase by T6P.

\section{Discussion}

Trehalose synthesis and hydrolysis have been established as an underlying molecular process for stress survival and adaptation to the environmental changes in S. cerevisiae and other microorganisms (Crowe et al., 1984; Hottiger et al., 1987; Singer and Lindquist, 1998). Discovered decades ago, its relationship with key biochemical processes involving carbohydrate and energy metabolism has not been clearly elucidated yet (Blomberg, 2000; François and Parrou, 2001; Gancedo and Flores, 2004; Noubhani et al., 2009; Thevelein and Hohmann, 1995).

There is a need to further develop models of cellular metabolic processes and to analyze those with new tools and approaches derived from the systems biology perspective. By admitting that metabolism is truly a systemic process, one may begin to understand its emergent behavior as more than the sum of its constituent parts. Moreover, through providing a theoretical framework to which the vast array of available metabolic data may be fused, one may begin to uncover the nonlinear interactions that govern its complexities.

In the case of trehalose metabolism, a theoretical approach has got us some way to understanding the interactive dynamics of the pathway. In this chapter, we demonstrate how to generate a kinetic model and use it to investigate the effects of the heat shock and TPS1 mutation on trehalose 
metabolism. In the case of heat shock, the calculated increase in concentrations of intermediate metabolites reflects the previously measured elevation of glucose uptake and enzyme activities of trehalose metabolism (Ribeiro et al., 1994). Moreover, the large response of trehalose concentration supports experimental observations (Hottiger et al., 1987; Ribeiro et al., 1994). Intriguingly, the key intermediate at a crossroad of glycolysis, pentose phosphate, glycogen, and trehalose pathways- G6P-is also greatly elevated, suggesting its critical role in stress response.

The use of our model to investigate the effect of TPS1 mutation showed an expected increase in the concentration of metabolites upstream to T6P, that is, G6P and UDP glucose and a decrease in the concentration of downstream derivatives, that is, T6P, trehalose, and glucose. In addition, our model suggests that the intracellular concentration of T6P is insufficient to reduce significantly the hexokinase activity, as it has been postulated previously (Blázquez et al., 1993).

Due to the scope of this chapter, we limit ourselves to the kinetic description of a single pathway. Nevertheless, it would be an intriguing topic of future research to combine the model constructed here with a kinetic model of glycolysis, as there is a direct link through hexokinase and carbohydrate intermediates such as G6P and/or to extend the model to include glycogen (interesting for the TPS1 mutant). Another interesting extension of the model would be the addition of phosphate dynamics, which could influence the concentration of inorganic phosphate (inhibitor of T6P synthase) and the consumption and regeneration of ATP in the cell.

In this chapter, we have presented the necessary steps for constructing a kinetic model of a metabolic pathway, and examples of the kinds of analyses that can be performed with such a model. Application of such methodologies can help us decipher metabolic behavior, especially when combined with laboratory experiments that will test and verify the simulation results, and suggest new directions and hypotheses for the study of the pathway of interest.

\section{ACKNOWLEDGMENTS}

We are grateful for the financial support of the BBSRC and EPSRC through grant BB/ C008219/1 “The Manchester Centre for Integrative Systems Biology (MCISB).” We also thank Michael Howard for his invaluable oversight, and our MCISB colleagues.

\section{REFERENCES}

Bell, W., et al. (1998). Composition and functional analysis of the Saccharomyces cerevisiae trehalose synthase complex. J. Biol. Chem. 273, 33311-33319.

Berry, D. B., and Gasch, A. P. (2008). Stress-activated genomic expression changes serve a preparative role for impending stress in yeast. Mol. Biol. Cell 19, 4580-4587. 
Blázquez, M. A., et al. (1993). Trehalose-6-phosphate, a new regulator of yeast glycolysis that inhibits hexokinases. FEBS Lett. 329, 51-54.

Blomberg, A. (2000). Metabolic surprises in Saccharomyces cerevisiae during adaptation to saline conditions: Questions, some answers and a model. FEMS Microbiol. Lett. 182, 1-8.

Cabib, E., and Leloir, L. F. (1958). Biosynthesis of trehalose phosphate. J. Biol. Chem. 231, 259-275.

Canelas, A. B., et al. (2009). Quantitative evaluation of intracellular metabolite extraction techniques for yeast metabolomics. Anal. Chem. 81, 7379-7389.

Covert, M. W., Famili, I., and Palsson, B.Ø. (2003). Identifying constraints that govern cell behavior: A key to converting conceptual to computational models in biology? Biotechnol. Bioeng. 84, 763-772.

Crowe, J. H., Crowe, L. M., and Chapman, D. (1984). Preservation of membranes in anhydrobiotic organisms-The role of trehalose. Science 223, 701-703.

Daugherty, J. P., Kraemer, W. F., and Joshi, J. G. (1975). Purification and properties of phosphoglucomutase from Fleischmann's yeast. Eur. J. Biochem. 57, 115-126.

De Mesquita, J. F., Panek, A. D., and de Araujo, P. S. (2003). In silico and in vivo analysis reveal a novel gene in Saccharomyces cerevisiae trehalose metabolism. BMC Genomics 4, 45.

Dobson, P. D., et al. (2010). Further developments towards a genome-scale metabolic model of yeast. BMC Syst. Biol. 4, 145.

François, J., and Parrou, J. L. (2001). Reserve carbohydrates metabolism in the yeast Saccharomyces cerevisiae. FEMS Microbiol. Rev. 25, 125-145.

Gancedo, C., and Flores, C. L. (2004). The importance of a functional trehalose biosynthetic pathway for the life of yeasts and fungi. FEMS Yeast Res. 4, 351-359.

Gasch, A. P., et al. (2000). Genomic expression programs in the response of yeast cells to environmental changes. Mol. Biol. Cell 11, 4241-4257.

Gelperin, D. M., et al. (2005). Biochemical and genetic analysis of the yeast proteome with a movable ORF collection. Genes Dev. 19, 2816-2826.

Guranowski, A., et al. (2004). Uridine 5'-polyphosphates (p(4)U and $\mathrm{p}(5) \mathrm{U})$ and uridine(5') polyphospho(5')nucleosides (Up(n)Ns) can be synthesized by UTP: Glucose-1-phosphate uridylyltransferase from Saccharomyces cerevisiae. FEBS Lett. 561, 83-88.

Hatzimanikatis, V., and Bailey, J. E. (1997). Effects of spatiotemporal variations on metabolic control: Approximate analysis using (log)linear kinetic models. Biotechnol. Bioeng. 54, 91-104.

Hottiger, T., Schmutz, P., and Wiemken, A. (1987). Heat-induced accumulation and futile cycling of trehalose in Saccharomyces cerevisiae. J. Bacteriol. 169, 5518-5522.

Hucka, M., et al. (2003). The Systems Biology Markup Language (SBML): A medium for representation and exchange of biochemical network models. Bioinformatics 19, 524-531.

Kauffman, K. J., Prakash, P., and Edwards, J. S. (2003). Advances in flux balance analysis. Curr. Opin. Biotechnol. 14, 491-496.

Klipp, E., et al. (2005). Systems Biology in Practice: Concepts, Implementation and Application. Wiley-VCH, Weinheim.

Krause, F., et al. (2011). Sustainable modelling in systems biology: The roles of standards and semantic annotations. Methods Enzymol. 500, (this issue).

Lazebnik, Y. (2002). Can a biologist fix a radio? Or, what I learned while studying apoptosis. Cancer Cell 2, 179-182.

Le Novère, N., et al. (2005). Minimum Information Required In the Annotation of Models (MIRIAM). Nat. Biotechnol. 23, 1509-1515.

Le Novère, N., et al. (2009). The systems biology graphical notation. Nat. Biotechnol. 27, 735-741.

Li, C., et al. (2010). BioModels Database: An enhanced, curated and annotated resource for published quantitative kinetic models. BMC Syst. Biol. 4, 92. 
Liebermeister, W., and Klipp, E. (2006). Bringing metabolic networks to life: Convenience rate law and thermodynamic constraints. Theor. Biol. Med. Model. 3, 41.

Malys, N., et al. (2004). The 'scavenger' $\mathrm{m} 7 \mathrm{G}$ pppX pyrophosphatase activity of Dcs1 modulates nutrient-induced responses in yeast. Nucleic Acids Res. 32, 3590-3600.

Malys, N., et al. (2011). Protein production in S. cerevisiae for systems biology studies. Methods Enzymol. 500, (in this volume).

Mendes, P., and Kell, D. (1998). Non-linear optimization of biochemical pathways: Applications to metabolic engineering and parameter estimation. Bioinformatics 14, 869-883.

Noubhani, A., et al. (2009). The trehalose pathway regulates mitochondrial respiratory chain content through hexokinase 2 and cAMP in Saccharomyces cerevisiae. J. Biol. Chem. 284, 27229-27234.

Palsson, B.Ø. (2006). Systems Biology: Properties of Reconstructed Networks. Cambridge University Press, Cambridge.

Price, N. D., Reed, J. L., and Palsson, B.Ø. (2004). Genome-scale models of microbial cells: Evaluating the consequences of constraints. Nat. Rev. Microbiol. 2, 886-897.

Pritchard, L., and Kell, D. B. (2002). Schemes of flux control in a model of Saccharomyces cerevisiae glycolysis. Eur. J. Biochem. 269, 3894-3904.

Ray, W. J., and Roscelli, G. A. (1964). Phosphoglucomutase pathway-Investigation of phospho-enzyme isomerization. J. Biol. Chem. 239, 3935.

Rep, M., et al. (2000). The transcriptional response of Saccharomyces cerevisiae to osmotic shock. Hot1p and Msn2p/Msn4p are required for the induction of subsets of high osmolarity glycerol pathway-dependent genes. J. Biol. Chem. 275, 8290-8300.

Ribeiro, M. J. S., Silva, J. T., and Panek, A. D. (1994). Trehalose metabolism in Saccharomyces cerevisiae during heat-shock. Biochim. Biophys. Acta 1200, 139-147.

Singer, M. A., and Lindquist, S. (1998). Multiple effects of trehalose on protein folding in vitro and in vivo. Mol. Cell 1, 639-648.

Smallbone, K., et al. (2007). Something from nothing-Bridging the gap between constraint-based and kinetic modelling. FEBS J. 274, 5576-5585.

Spasić, I., et al. (2009). KiPar, a tool for systematic information retrieval regarding parameters for kinetic modelling of yeast metabolic pathways. Bioinformatics 25, 1404-1411.

Szallasi, Z., Stelling, J., and Periwal, V. (2006). System Modeling in Cellular Biology: From Concepts to Nuts and Bolts. MIT Press, Boston.

Teusink, B., et al. (1998). The danger of metabolic pathways with turbo design. Trends Biochem. Sci. 23, 162-169.

Thevelein, J. M., and Hohmann, S. (1995). Trehalose synthase-Guard to the gate of glycolysis in yeast. Trends Biochem. Sci. 20, 3-10.

Vandercammen, A., François, J., and Hers, H. G. (1989). Characterization of trehalose6-phosphate synthase and trehalose-6-phosphate phosphatase of Saccharomyces cerevisiae. Eur. J. Biochem. 182, 613-620.

Villéger, A. C., Pettifer, S. R., and Kell, D. B. (2010). Arcadia: A visualization tool for metabolic pathways. Bioinformatics 26, 1470-1471.

Voit, E. O. (1991). Canonical Nonlinear Modeling: S-system Approach to Understanding Complexity. Van Nostrand Reinhold, New York.

Voit, E. O. (2003). Biochemical and genomic regulation of the trehalose cycle in yeast: Review of observations and canonical model analysis. J. Theor. Biol. 223, 55-78.

Westerhoff, H. V., and van Dam, J. C. (1987). Mosaic Nonequilibrium Thermodynamics and Control of Biological Free-Energy Transduction. Elsevier, Amsterdam.

Wiechert, W. (2002). Modeling and simulation: Tools for metabolic engineering. J. Biotechnol. 94, 37-63.

Wright, B. E., Tai, A., and Killick, K. A. (1977). 4th expansion and glucose perturbation of dictyostelium kinetic-model. Eur. J. Biochem. 74, 217-225. 